\title{
Most Intense X-Ray Lines of the Helium Isoelectronic Sequence for Plasmas Diagnostic
}

\author{
Ibrahima Sakho* \\ Department of Experimental Sciences, UFR Sciences and Technologies, University of Thies, Thies, Senegal \\ Email: *aminafatima_sakho@yahoo.fr
}

How to cite this paper: Sakho, I. (2020) Most Intense X-Ray Lines of the Helium Isoelectronic Sequence for Plasmas Diagnostic. Journal of Modern Physics, 11, 487-501.

https://doi.org/10.4236/jmp.2020.114031

Received: January 23, 2020

Accepted: March 23, 2020

Published: March 26, 2020

Copyright (c) 2020 by author(s) and Scientific Research Publishing Inc. This work is licensed under the Creative Commons Attribution International License (CC BY 4.0).

http://creativecommons.org/licenses/by/4.0/

\section{(c) (i) Open Access}

\begin{abstract}
We report accurate wavelengths for the three most intense lines (resonance line: $1 \mathrm{~s}^{2} \mathrm{I} 0-1 \mathrm{~s} 2 \mathrm{p}{ }^{1} \mathrm{P} 1$, intercombination line: $1 \mathrm{~s}^{2}{ }^{1} \mathrm{~S} 0-1 \mathrm{~s} 2 \mathrm{p}{ }^{3} \mathrm{P} 1$ and forbidden line: $1 s^{2}{ }^{1} \mathrm{~S} 0-1 \mathrm{~s} 2 \mathrm{~s}^{3} \mathrm{~S} 1$ ) along with wavelengths for the $1 \mathrm{~s}^{2}{ }^{1} \mathrm{~S} 0-1 \mathrm{~s} n \mathrm{p}^{1} \mathrm{P} 1$ and ${ }^{1} \mathrm{~S} 0-1 \operatorname{s} n \mathrm{p}^{3} \mathrm{P} 2(2 \leq n \leq 25)$ transitions in He-like systems $(Z=2-13)$. The first spectral lines that belong to the above transitions are established in the framework of the Screening Constant per Unit Nuclear Charge method. The results obtained agree excellently with various experimental and theoretical literature data. The uncertainties in wavelengths between the present calculations and the available literature data are less than $0.004 \AA$. A host of new data listed in this paper may be of interest in astrophysical and laboratory plasmas diagnostic.
\end{abstract}

\section{Keywords}

He-Like Systems, Semi-Empirical, Screening Constant Per Unit Nuclear Charge, Excited States, X-Ray Spectra

\section{Introduction}

The helium-like isoelectronic series emit strong X-ray wavelengths. The most intense lines of these systems are the resonance line designated by $\omega$ (also labelled $r .1 \mathrm{~s}^{2}{ }^{1} \mathrm{~S}_{0}-1 \mathrm{~s} 2 \mathrm{p}^{1} \mathrm{P}_{1}$ ), the intercombination lines $(x+y)$ (or $i: 1 \mathrm{~s}^{2}{ }^{1} \mathrm{~S}_{0}$ $\left.1 \mathrm{~s} 2 \mathrm{p}^{3} \mathrm{P}_{2,1}\right)$ and the forbidden line $z\left(\right.$ or $f: 1 \mathrm{~s}^{2} \mathrm{~S}_{0}-1 \mathrm{~s} 2 \mathrm{~s}{ }^{3} \mathrm{~S}_{1}$ ). These three lines correspond to the transitions between the $n=2$ excited shell and the $n=1$ ground state shell. The determination of these lines is of great interest because the line ratios $f / i$ and $(f+i) / r$ provided respectively electrons density $\left(n_{e} \sim 10^{8}-10^{13}\right.$ $\left.\mathrm{cm}^{-3}\right)$ and electrons temperature $\left(T_{e} \sim 1-10 \mathrm{MK}\right)$ as first shown by Gabriel and Jordan [1] and are widely used for collisional solar plasma diagnostics [1] [2] [3]. On the other hand, these line ratios enable also to determine the prevailed ioni- 
zation processes (photo-ionization and/or collisional ionization) in the plasma [4] [5] [6]. Traditionally, He-like ions $f / i$ line ratios have been used to derive electron densities of X-ray line-emitting regions since the populations of the $2^{3} \mathrm{P}$ level are controlled by collisional excitation from the $2^{3} \mathrm{~S}$ level [1]. At low density, the $n=2$ states are populated by electron excitation and then decay radiatively. Then, the relative intensities of the three lines are independent of the density [7]. Above the critical density $\left(n_{\text {crit }}\right):\left(n_{\text {crit }} C \sim A, C\right.$ being the rate coefficient for collisional excitation $2^{3} S \rightarrow 2^{3} P$ and $A$ denotes the radiative transition probability of $2^{3} S \rightarrow 1^{1} S$ ), the $2^{3} S$ upper level of the forbidden line becomes to be depleted by collision to the $2^{3} \mathrm{P}$ upper levels of the intercombination line. As a result, when the electron density increases, the intensity of the forbidden line decreases strongly whereas that of the intercombination line increases. However, in the case of a strong UV radiation, the photo-excitation $2^{3} \mathrm{~S} \rightarrow 2^{3} \mathrm{P}$ becomes no negligible. Subsequently, the ratio $(f i)$ of the forbidden line on the intercombination line is no longer an electron density diagnostic. As concern the ratio $(f+$ i) $/ r$, it is sensitive to electron temperature as the dependence of the collisional excitation rates with the temperature for the resonance line is not the same for the forbidden and intercombination lines. In short, for plasma dominated by photo-ionization and recombination, the forbidden line (or the intercombination line at high density) becomes much stronger than the resonance line. In the case of plasma dominated by collisional ionization and excitation, the resonance line is stronger or comparable to the forbidden line and the intercombination line [4]. The following considerations indicate that the determination of the most intensive lines of Helium-like ions in the $\mathrm{X}$-ray range is of great interest in laboratory and astrophysical plasma diagnostics.

On the experimental side, high-precision measurements of the energy difference between $\mathrm{S}$ and $\mathrm{P}$ levels in the helium isoelectronic series were made three decades ago. Robinson [7] presents measurements of the $1 s^{2}{ }^{1} S_{0}-1 \mathrm{~s} n \mathrm{p}{ }^{1} P_{1}$ series of the Helium isoelectronic sequence for Be III, B IV and C V. Since that time, many experiments have been improved. Twelve lines in the region $20-100 \AA$ belonging to the resonance series of Be III, B IV, C V and O VII are remeasured by Svensson [8] using spectrograms. Beiersdorfer et al. [9] use the tokamak plasmas from the Princeton Large Torus (PLT) high-resolution Johann spectrometer to report the $n=2 \rightarrow 1 \mathrm{X}$-ray transitions of Helium-like potassium, scandium, titanium, vanadium, chromium, and iron $(Z=19-26)$ along with wavelengths belonging to the $1 s^{2}{ }^{1} S_{0}-1 s n p{ }^{1} P_{1}(n=3-5)$ transitions. Furthermore, Engtröm and Litzén [10] generate spectra of $\mathrm{C}, \mathrm{N}$ and $\mathrm{O}$ simultaneously by focusing $1 \mathrm{GW}$ laser pulses on targets made of either ammonium hydrogen carbonate or ammonium oxalate and then determine the wavelengths of the $1 \mathrm{~s}^{2} \mathrm{~S}-1 \operatorname{snp}{ }^{1} \mathrm{P}(n=2-4)$ resonance lines in N VI and O VII (17 - $30 \AA \AA \AA)$ ) with uncertainties ranging from 0.2 to 0.7 $\mathrm{m} \AA \AA \AA$. Bartnik et al. [11] measure the wavelengths of the $1 \mathrm{~s} n \mathrm{p}{ }^{1} \mathrm{P}-1 \mathrm{~s}^{2}{ }^{1} \mathrm{~S}(n=4-$ 10) transitions in He-like O VII in laser-produced gas puff plasmas with an accuracy measurement ranging between (1.5 - $3.0 \mathrm{~m} \AA$ ).

On the theoretical side, many techniques are presented. Acaad et al. [12] con- 
struct wave function expanded in a triple series of Laguerre polynomials of the perimertric coordinates to study the $\mathrm{S}$ and $\mathrm{P}$ states of the helium isoelectronic sequence and report nonrelativistic wavelengths and total wavelengths including mass polarization relativistic and, the Lamb shift corrections for $Z=2-9$ belonging to the $1 \mathrm{~s} n \mathrm{p}{ }^{1} \mathrm{P}-1 \mathrm{~s}^{21} \mathrm{~S}(n=2-5)$ transitions. In addition, Safronova et al. [13] apply the MZ code through a perturbation theory based on hydrogen-like functions to compute wavelengths of highly charged He-like ions $(Z=6-54)$ for both satellite lines ( 1 s $\left.2 P n l-1 s^{2} n^{\prime} l, n, n^{\prime}=2,3\right)$ and $\left(1 s n p^{1,3} \mathrm{P}-1 s^{2}, n=2,3\right.$ and $1 \mathrm{~s} 2 \mathrm{~s}^{1,3} \mathrm{~S}-1 \mathrm{~s}^{2}$ ) transitions. Additionally, the plasma simulation code CLOUDY is used by Porter [14] to present wavelengths of the UV, intercombination, forbidden, and resonance transitions oh He-like ions for $Z=6-14$ and for $Z=16,18$, 20 , and 26. But, as far as we know, the wavelengths cannot be directly determined within a single analytical formula for a whole members of He-like ions using one of the preceding method or one of the other existing computational techniques. Then, analytical spectral lines in two-electron systems such as the Balmer or the Lyman spectral lines of the hydrogen-like systems are not yet established. In this paper, we intend to present analytical spectral lines belonging to the resonance line: $1 \mathrm{~s}^{2}{ }^{1} \mathrm{~S}_{0}-1 \mathrm{~s} 2 \mathrm{p}{ }^{1} \mathrm{P}_{1}$ and intercombination line: $1 \mathrm{~s}^{2}{ }^{1} \mathrm{~S}_{0}-1 \mathrm{~s} 2 \mathrm{p}$ ${ }^{3} \mathrm{P}_{2,1}$ along the $1 \mathrm{~s}^{2} \mathrm{~S}_{0}-1 \mathrm{~s} n \mathrm{p}{ }^{1} \mathrm{P}_{1}(n \leq 10)$ transitions in the helium isoelectronic sequence. In our study, we use the Screening Constant per Unit Nuclear Charge (SCUNC) method suitable in the analysis of atomic spectra [15] [16]. All the results obtained in the present work compared very well to the available experimental and theoretical literature data. A host of data listed in this paper may be of interest in astrophysical and laboratory plasmas diagnostic.

In section 2, we present the theoretical procedure adopted in this work. In section 3, the presentation and the discussion of the results are made. A comparison of our results with available experimental and theoretical results is also made.

\section{Theory}

\subsection{Brief Description of the SCUNC Formalism}

In the framework of Screening Constant per Unit Nuclear Charge formalism, total energy of $\left(N \ell, n \ell^{\prime}\right)^{2 S+1} L^{\pi}$ excited states are expressed in the form (in rydberg units)

$$
E\left(N \ell n \ell^{\prime} ;{ }^{2 S+1} L^{\pi}\right)=-Z^{2}\left(\frac{1}{N^{2}}+\frac{1}{n^{2}}\left[1-\beta\left(N \ell n \ell^{\prime} ;{ }^{2 S+1} L^{\pi} ; Z\right)\right]^{2}\right) .
$$

In this equation, the principal quantum numbers $N$ and $n$, are respectively for the inner and the outer electron of He-isoelectronic series. In this equation, the $\beta$-parameters are screening constant by unit nuclear charge expanded in inverse powers of $Z$ and given by

$$
\beta\left(N \ell n \ell^{\prime} ;{ }^{2 S+1} L^{\pi} ; Z\right)=\sum_{k=1}^{q} f_{k} \times\left(\frac{1}{Z}\right)^{k} .
$$

where $f_{k}=f_{k}\left(N \ell n \ell^{\prime} ;{ }^{2 S+1} L^{\pi}\right)$ are parameters to be evaluated empirically. 


\subsection{Energies for the Ground State}

For the ground state, Equations (1) and (2) give

$$
E\left(1 s^{2} ;{ }^{1} S_{0}\right)=-Z^{2}\left(1+\left\{1-\frac{f_{1}}{Z}-\frac{f_{2}}{Z^{2}}-\frac{f_{3}}{Z^{3}}\right\}^{2}\right) .
$$

Using the experimental total energy of $\mathrm{He}$ I, Li II and Be III respectively (in eV) -79.01 [17], -198.09 [18] and -371.60 [18], the screening constants in Equation (4) are evaluated by use of the infinite rydberg energy $1 \mathrm{Ryd}=13.605698$ $\mathrm{eV}$. We find then

$$
E\left(1 s^{2} ;{ }^{1} S_{0}\right)=-Z^{2}\left(1+\left\{1-\frac{0.625085938}{Z}-\frac{0.031315676}{Z^{2}}-\frac{0.059849712}{Z^{3}}\right\}^{2}\right) \text {. }
$$

\subsection{Spectral Lines of the ${ }^{1}{ }^{1} S_{0}-1 s 2 p{ }^{1} P_{1}$ Resonance Transition}

During the $1 \mathrm{~s}^{2} \mathrm{~S}_{0}-1 \mathrm{~s} 2 \mathrm{p}^{1,3} \mathrm{P}_{1}$ transitions, the energy of the system varies as

$$
\Delta E=\frac{h c}{\lambda}=E\left(1 s 2 p ;{ }^{1,3} P_{1}\right)-E\left(1 s^{2} ;{ }^{1} S_{0}\right) .
$$

Using Equations (1) and (3b), we obtain from Equation (4)

For $2 \leq Z \leq 15$

$$
\begin{aligned}
\frac{h c}{\lambda}= & Z^{2}\left(1+\left\{1-\frac{0.625085938}{Z}-\frac{0.031315676}{Z^{2}}-\frac{0.059849712}{Z^{3}}\right\}^{2}\right) \\
& -Z^{2}\left(1+\frac{1}{4}\left\{1-\frac{f_{1}}{Z}-\frac{f_{2} \times\left(Z-Z_{0}\right)}{Z^{2}}-\frac{f_{1}^{2} \times\left(Z-Z_{0}\right)^{2} \times\left(Z-Z_{0}^{\prime}\right)}{Z^{3}}\right.\right. \\
& \left.\left.-\frac{f_{1}^{2} \times\left(Z-Z_{0}\right)^{2} \times\left(Z-Z_{0}^{\prime}\right)^{2}}{Z^{4}}-\frac{f_{1} \times\left(Z-Z_{0}\right)^{2} \times\left(Z-Z_{0}^{\prime}\right)^{2}}{Z^{5}}\right\}^{2}\right)
\end{aligned}
$$

In these equations, $Z_{0}$ and $Z_{0}^{\prime}$ denote the nuclear charge of the helium-like systems used in the empirical determination of the $f_{i}^{\prime}$-screening constants. On the basis of $h=6.626276 \times 10^{-34} \mathrm{~J} \cdot \mathrm{s}, c=2.99792458 \times 10^{8} \mathrm{~m} / \mathrm{s}$ and $e=1.602189 \times 10^{-19} \mathrm{C}$ and using for $1 s^{2}{ }^{1} \mathrm{~S}_{0}-1 \mathrm{~s} 2 p^{3} \mathrm{P}_{1}$ the experimental wavelengths of $\mathrm{He} \mathrm{I}\left(Z_{0}=2\right)$ and that of $\mathrm{Li}$ II $\left(Z_{0}^{\prime}=3\right)$ respectively $584.3339 \AA$ [19] and 199.280 ̊ [7], Equation (5a) gives $f_{1}=1.004778731$ and $f_{2}=0.026277861$. We obtain then explicitly

$$
\begin{aligned}
\frac{1}{\lambda}= & Z^{2}\left(1+\left\{1-\frac{0.625085938}{Z}-\frac{0.031315676}{Z^{2}}-\frac{0.059849712}{Z^{3}}\right\}^{2}\right) \\
& -Z^{2}\left(1+\frac{1}{4}\left\{1-1.004778731 \frac{1}{Z}-0.026277861 \frac{Z-2}{Z^{2}}\right.\right. \\
& -0.000690525 \frac{(Z-2)^{2} \times(Z-3)}{Z^{3}}-0.000690525 \frac{(Z-2)^{2} \times(Z-3)^{2}}{Z^{4}} \\
& \left.\left.-0.026277861 \frac{(Z-2)^{2} \times(Z-3)^{2}}{Z^{5}}\right\}^{2}\right) \times 10973644.9
\end{aligned}
$$


In Equation (5b), wavelengths are expressed in meters $(m)$ and the infinite rydberg energy $1 \mathrm{Ryd}=13.605698 \mathrm{eV}$ is used along with $1 \mathrm{eV}=1.602189 \times 10^{-19}$ J. So $\operatorname{Ryd} / h c=10973644.9(m)$.

\subsection{Spectral Lines of the $1 s^{2}{ }^{1} S_{0}-1 s 2 p^{3} P_{1}$ Intercombination Transition}

Using Equations (1) and (3b), Equation (4) yields for the $1 \mathrm{~s}^{2}{ }^{1} \mathrm{~S}_{0}-1 \mathrm{~s} 2 \mathrm{p}{ }^{3} \mathrm{P}_{1}$ intercombination transition

$$
\begin{aligned}
\frac{h c}{\lambda}= & Z^{2}\left(1+\left\{1-\frac{0.625085938}{Z}-\frac{0.031315676}{Z^{2}}-\frac{0.059849712}{Z^{3}}\right\}^{2}\right) \\
& -Z^{2}\left(1+\frac{1}{4}\left\{1-\frac{f_{1}^{\prime \prime}}{Z}-\frac{f_{2}^{\prime \prime} \times\left(Z-Z_{0}\right)}{Z^{2}}-\frac{f_{2}^{\prime \prime 2} \times\left(Z-Z_{0}\right) \times\left(Z-Z_{0}^{\prime}\right)^{2}}{Z^{3}}\right.\right. \\
& \left.\left.-\frac{f_{2}^{\prime \prime 2} \times\left(Z-Z_{0}\right)^{2} \times\left(Z-Z_{0}^{\prime}\right)^{2}}{Z^{4}}-\frac{f_{2}^{\prime \prime 2} \times\left(Z-Z_{0}\right)^{2} \times\left(Z-Z_{0}^{\prime}\right)^{3}}{Z^{5}}\right\}^{2}\right)
\end{aligned}
$$

Here again, $Z_{0}$ and $Z_{0}^{\prime}$ denote the nuclear charge of the helium-like systems used in the empirical determination of the $f_{i}^{\prime \prime}$-parameters. For $1 \mathrm{~s}^{2}{ }^{1} \mathrm{~S}_{0}-1 \mathrm{~s} 2 \mathrm{p}^{3} \mathrm{P}_{1}$, the experimental wavelengths of $\mathrm{He} \mathrm{I}\left(Z_{0}=2\right)$ and that of B IV $\left(Z_{0}^{\prime}=5\right)$ are respectively equal to 591.4121 [19] and $61.0880 \AA$ [9] as quoted in Ref. [12], we obtain from Equation (6a) $f_{1}^{\prime \prime}=0.967951498$ and $f_{2}=-0.06781546$.

Equation (6a) becomes then

$$
\begin{aligned}
\frac{1}{\lambda}= & Z^{2}\left(1+\left\{1-\frac{0.625085938}{Z}-\frac{0.031315676}{Z^{2}}-\frac{0.059849712}{Z^{3}}\right\}^{2}\right) \\
& -Z^{2}\left(1+\frac{1}{4}\left\{1-0.967951498 \frac{1}{Z}+0.06781546 \frac{Z-2}{Z^{2}}\right.\right. \\
& -0.004598936 \frac{(Z-2) \times(Z-5)^{2}}{Z^{3}}-0.004598936 \frac{(Z-2)^{2} \times(Z-5)^{2}}{Z^{4}} \\
& \left.\left.-0.004598936 \frac{(Z-2)^{2} \times(Z-5)^{3}}{Z^{5}}\right\}^{2}\right) \times 10973644.9
\end{aligned}
$$

\subsection{Spectral Lines of the $1 s^{2}{ }^{1} S_{0}-1 s 2 s{ }^{3} S_{1}$ Forbidden Transitions}

For the $1 \mathrm{~s}^{2} \mathrm{~S}_{0}-1 \mathrm{~s} 2 \mathrm{~s}^{3} \mathrm{~S}_{1}$ forbidden transitions, the spectral lines are given by

$$
\begin{aligned}
\frac{h c}{\lambda}= & Z^{2}\left(1+\left\{1-\frac{0.625085938}{Z}-\frac{0.031315676}{Z^{2}}-\frac{0.059849712}{Z^{3}}\right\}^{2}\right) \\
& -Z^{2}\left(1+\frac{1}{4}\left\{1-\frac{f_{1}^{\prime \prime}}{Z}-\frac{f_{2}^{\prime \prime} \times\left(Z-Z_{0}\right)}{Z^{2}}-\frac{f_{2}^{\prime \prime 2} \times\left(Z-Z_{0}\right) \times\left(Z-Z_{0}^{\prime}\right)}{Z^{3}}\right.\right. \\
& -\frac{f_{2}^{\prime \prime 2} \times\left(Z-Z_{0}\right)^{2} \times\left(Z-Z_{0}^{\prime}\right)}{Z^{4}}-\frac{f_{2}^{\prime \prime 2} \times\left(Z-Z_{0}\right)^{2} \times\left(Z-Z_{0}^{\prime}\right)^{3}}{Z^{5}} \\
& \left.\left.-\frac{f_{2}^{\prime \prime 2} \times\left(Z-Z_{0}\right) \times\left(Z-Z_{0}^{\prime}\right)^{3}}{Z^{6}}\right\}^{2}\right)
\end{aligned}
$$


For $1 \mathrm{~s}^{2}{ }^{1} \mathrm{~S}_{0}-1 \mathrm{~s} 2 \mathrm{~s}{ }^{3} \mathrm{~S}_{1}$, the experimental wavelengths from NIST [20] for He I $\left(Z_{0}=2\right)$ and for Li II $\left(Z_{0}^{\prime}=3\right)$ are respectively equal to $625.563 \AA$ and $210.069 \AA$. Equation (7a) provides then $f_{1}^{\prime \prime}=0.816109425$ and $f_{2}^{\prime \prime}=-0.079252785$. Equation (7a) becomes explicitly

$$
\begin{aligned}
\frac{h c}{\lambda}= & Z^{2}\left(1+\left\{1-\frac{0.625085938}{Z}-\frac{0.031315676}{Z^{2}}-\frac{0.059849712}{Z^{3}}\right\}^{2}\right) \\
& -Z^{2}\left(1+\frac{1}{4}\left\{1-\frac{0.816109425}{Z}-\frac{0.079252785 \times(Z-2)}{Z^{2}}\right.\right. \\
& -\frac{0.006281003 \times(Z-2) \times(Z-3)}{Z^{3}}-\frac{0.006281003 \times(Z-2) \times(Z-3)}{Z^{3}} \\
& -\frac{0.006281003 \times(Z-2)^{2} \times(Z-3)^{2}}{Z^{4}}-\frac{0.006281003 \times(Z-2)^{2} \times(Z-3)^{3}}{Z^{5}} \\
& \left.\left.-\frac{0.006281003 \times(Z-2) \times(Z-3)^{3}}{Z^{6}}\right\}^{2}\right) \times 10973644.9
\end{aligned}
$$

\subsection{Spectral Lines of the $1 s^{2}{ }^{1} \mathrm{~S}_{0}-1 s n p^{1} \mathrm{P}_{1}$ Transitions}

Following the same reasoning above, we express from Equations (1) and (2) total energies belonging to the $1 \operatorname{sn} p^{1} \mathrm{P}_{1}$ levels

$$
\begin{aligned}
E\left(1 \text { snp } ;{ }^{1} P_{1}\right)= & -Z^{2}\left(1+\frac{1}{n^{2}}\left\{1-\frac{f_{1}}{Z(n-1)}-\frac{f_{2}}{Z}-\frac{f_{3} \times\left(Z-Z_{0}\right)}{Z^{2} n^{2}}\right.\right. \\
& \left.\left.-\frac{f_{3} \times\left(Z-Z_{0}\right)^{2} \times\left(Z-Z_{0}^{\prime}\right)}{Z^{3}}-\frac{f_{3} \times\left(Z-Z_{0}\right)^{2} \times\left(Z-Z_{0}^{\prime}\right)^{2}}{Z^{4}}\right\}^{2}\right)
\end{aligned}
$$

For the $1 s^{2} \mathrm{~S}_{0}-1 \mathrm{~s} n \mathrm{p}^{1} \mathrm{P}_{1}$ transitions, we get

$$
\begin{aligned}
\frac{h c}{\lambda}= & Z^{2}\left(1+\left\{1-\frac{0.625085938}{Z}-\frac{0.031315676}{Z^{2}}-\frac{0.059849712}{Z^{3}}\right\}^{2}\right) \\
& -Z^{2}\left(1+\frac{1}{n^{2}}\left\{1-\frac{f_{1}}{Z(n-1)}-\frac{f_{2}}{Z}-\frac{f_{3} \times\left(Z-Z_{0}\right)}{Z^{2} n^{2}}\right.\right. \\
& \left.\left.-\frac{f_{3} \times\left(Z-Z_{0}\right)^{2} \times\left(Z-Z_{0}^{\prime}\right)}{Z^{3}}-\frac{f_{3} \times\left(Z-Z_{0}\right)^{2} \times\left(Z-Z_{0}^{\prime}\right)^{2}}{Z^{4}}\right\}^{2}\right)
\end{aligned}
$$

For $1 \mathrm{~s}^{2} \mathrm{~S}_{0}-1 \mathrm{~s} 3 \mathrm{p}{ }^{3} \mathrm{P}_{1}$ and $1 \mathrm{~s}^{2}{ }^{1} \mathrm{~S}_{0}-1 \mathrm{~s} 4 \mathrm{p}{ }^{3} \mathrm{P}_{1}$ transitions, the corresponding experimental wavelengths of $\mathrm{Li}$ II $\left(Z_{0}=3\right)$ are respectively equal to $178.014 \AA$ and $171.575 \AA$ [7]. In addition, for Be III $\left(Z_{0}^{\prime}=4\right)$, the wavelength for to the $1 \mathrm{~s}^{2} \mathrm{~S}_{0}$ $1 \mathrm{~s} 3 \mathrm{p}^{3} \mathrm{P}_{1}$ transition is $88.314 \AA$ [7]. Using these wavelengths, we get from Equation (8b) $f_{1}=0.011679205, f_{2}=1.003675341$, and $f_{3}=0.008177868$. The spectral lines belonging to the $1 \mathrm{~s}^{2} \mathrm{~S}_{0}-1 \mathrm{~s} n \mathrm{p}^{1} \mathrm{P}_{1}$ transitions is then in the shape. 


$$
\begin{aligned}
\frac{1}{\lambda}= & Z^{2}\left(1+\left\{1-\frac{0.625085938}{Z}-\frac{0.031315676}{Z^{2}}-\frac{0.059849712}{Z^{3}}\right\}^{2}\right) \\
& -Z^{2}\left(1+\frac{1}{n^{2}}\left\{1-0.011679205 \frac{1}{Z(n-1)}-1.003675341 \frac{1}{Z}\right.\right. \\
& -0.008177868 \frac{Z-3}{Z^{2} n^{2}}-0.008177868 \frac{(Z-3)^{2} \times(Z-4)}{Z^{3}} \\
& \left.\left.-0.008177868 \frac{(Z-3)^{2} \times(Z-4)^{2}}{Z^{4}}\right\}^{2}\right) \times 10973644.9
\end{aligned}
$$

Before presenting and discussing the results obtained in this work, let us first move on explaining how electron-electrons and relativistic effects are accounted in the present SCUNC formalism. As mentioned previously [16] in the framework of the SCUNC formalism, all the relativistic corrections in many electron systems are incorporated in the $\beta$-parameters. To enlighten this point, let us move on considering the main relativistic terms in the Hamiltonian operator of $Q$-electron systems. For $Q$-electron systems, the Hamiltonian can be expressed as follows

$$
H=H_{0}+W .
$$

In this expression, $H_{0}$ denotes the nonrelativistic Hamiltonian and $W$ is the sum of the perturbation operators which includes mainly correction to kinetic energy $\left(W_{\text {kin }}\right)$, the Darwin term $\left(W_{\mathrm{D}}\right)$, mass polarization $\left(W_{\mathrm{M}}\right)$, spin-orbit corrections $\left(W_{\mathrm{so}}\right)$, spin-other orbit corrections $\left(W_{\mathrm{soo}}\right)$ and spin-spin corrections $\left(W_{\mathrm{ss}}\right)$. For Q-electron systems, the non-relativistic Hamiltonian and the perturbation operators are explicitly the following

$$
\begin{gathered}
H_{0}=\sum_{i=1}^{Q}\left[-\frac{1}{2} \nabla_{i}^{2}-\frac{Z}{r_{i}}\right]+\sum_{\substack{i, j=1 \\
i \neq j}}^{Q} \frac{1}{r_{i j}} ; W_{\text {kin }}=-\frac{\alpha^{2}}{8} \sum_{i=1}^{Q} \boldsymbol{p}_{i}^{4} ; W_{\mathrm{D}}=\frac{3 \pi \alpha^{2}}{2} \sum_{i=1}^{Q} \delta\left(\boldsymbol{r}_{i}\right) . \\
W_{\mathrm{M}}=-\frac{1}{M} \sum_{\substack{i, j=1 \\
i \neq j}}^{Q} \nabla_{i} \cdot \nabla_{j} ; W_{\mathrm{so}}=\frac{Z}{2 c^{2}} \sum_{i=1}^{Q} \frac{\boldsymbol{I}_{i} \cdot \boldsymbol{s}_{i}}{r_{i}^{3}} ; \\
W_{\mathrm{soo}}=-\frac{1}{2 c^{2}} \sum_{\substack{i, j=1 \\
i \neq j}}^{Q}\left[\frac{1}{r_{i j}^{3}}\left(\boldsymbol{r}_{i}-\boldsymbol{r}_{j}\right) \times \boldsymbol{p}_{i}\right] \cdot\left(\boldsymbol{s}_{i}+2 \boldsymbol{s}_{j}\right) . \\
W_{\mathrm{ss}}=\frac{1}{c^{2}} \sum_{\substack{i, j=1 \\
j>i}}^{Q} \frac{1}{r_{i j}^{3}}\left[\boldsymbol{s}_{i} \cdot \boldsymbol{s}_{j}-\frac{3\left(\boldsymbol{s}_{i} \cdot \boldsymbol{r}_{i j}\right)\left(\boldsymbol{s}_{j} \cdot \boldsymbol{r}_{i j}\right)}{r_{i j}^{2}}\right] .
\end{gathered}
$$

In these expressions, $\alpha$ denotes the fine structure constant and $M$ is the nuclear mass of the $Q$-electron systems. The energy value of the Hamiltonian (9a) is in the form

$$
E=E_{0}+w .
$$

with

$$
w=\left\langle W_{\text {kin }}\right\rangle+\left\langle W_{\mathrm{D}}\right\rangle+\left\langle W_{\mathrm{M}}\right\rangle+\left\langle W_{\mathrm{so}}\right\rangle+\left\langle W_{\text {soo }}\right\rangle+\left\langle W_{\mathrm{ss}}\right\rangle .
$$


For a-given $N l_{1}, n l_{2}$ configuration of He-like ions where $N, n$, and $l_{1}, l_{2}$, are respectively principal and orbital quantum numbers, the total energy is given by

$$
E=-\frac{Z^{2}}{N^{2}}-\frac{Z^{2}}{n^{2}}\left[1-\beta\left(N l_{1} n l_{2} ;{ }^{2 S+1} L^{\pi} ; Z\right)\right]^{2} .
$$

Developing Equation (9d), we obtain

$$
E=-\frac{Z^{2}}{N^{2}}-\frac{Z^{2}}{n^{2}}+\frac{Z^{2}}{n^{2}} \beta\left(N l_{1} n l_{2} ;{ }^{2 S+1} L^{\pi} ; Z\right)\left[2-\beta\left(N l_{1} n l_{2} ;{ }^{2 S+1} L^{\pi} ; Z\right)\right] .
$$

Equation (9e) can be rewritten in the form

$$
E=-\frac{Z^{2}}{N^{2}}-\frac{Z^{2}}{n^{2}}+\sum_{i=1}^{2} \frac{Z^{2}}{v_{i}^{2}} \beta_{i} \times\left[2-\beta_{i}\right] .
$$

This equation can be expressed in the same shape than Equation (9b)

$$
E=E_{0}+w .
$$

where

$$
\left\{\begin{array}{l}
E_{0}=-\frac{Z^{2}}{N^{2}}-\frac{Z^{2}}{n^{2}} \\
w=\sum_{i=1}^{2} \frac{Z^{2}}{v_{i}^{2}} \beta_{i} \times\left[2-\beta_{i}\right]
\end{array}\right.
$$

Using (9c) and the last equation in (10), we find

$$
\sum_{i=1}^{2} \frac{Z^{2}}{v_{i}^{2}} \beta_{i} \times\left[2-\beta_{i}\right]=\left\langle W_{\mathrm{kin}}\right\rangle+\left\langle W_{\mathrm{D}}\right\rangle+\left\langle W_{\mathrm{M}}\right\rangle+\left\langle W_{\mathrm{so}}\right\rangle+\left\langle W_{\mathrm{soo}}\right\rangle+\left\langle W_{\mathrm{ss}}\right\rangle .
$$

Equation (11) indicates clearly that, in the framework of the SCUNC-formalism, all the relativistic corrections are incorporated in the $\beta$-screening constants per unit nuclear charge. In the structure of the independent particles model disregarding all the relativistic effects, total energy is given by $E_{0}$. Subsequently $w=0$. This involves automatically $\beta=0$. Then, all relativistic effects are accounted implicitly in general Equation (1) via the $\beta$-parameters expanded in inverse powers

of $Z$ as shown by Equation (2) where the $f_{k}=f_{k}\left(N \operatorname{lnl}{ }^{\prime}{ }^{2 S+1} L^{\pi}\right)$-screening constants are evaluated empirically using experimental data incorporating all the relativistic effects and all electrons-electrons effects in many electron systems.

\section{Results and Discussions}

The present SCUNC wavelengths predictions for the wavelengths belonging to the $1 \mathrm{~s}^{2}{ }^{1} \mathrm{~S}_{0} \rightarrow 1 \mathrm{~s} n \mathrm{p}{ }^{1} \mathrm{P} 1(3 \leq n \leq 13)$ transitions in He-like $(\mathrm{Z}=3-38)$ ions are quoted in Table 1. Table 2 Presents a comparison between theoretical and experimental wavelengths of the $1{ }^{1} \mathrm{~S}_{0} \rightarrow n \mathrm{p}^{1} \mathrm{P}_{1}\left(1 \mathrm{~s}^{2} \mathrm{~S}_{0} \rightarrow 1 \mathrm{~s} n \mathrm{p}{ }^{1} \mathrm{P}_{1}\right)$ transitions of helium-like ions up to $Z=8$. The present SCUNC calculations values, are compared to the experimental data of Robinson [7], Svensson [8], Bartnik et al. [11] and to the experimental data of Engtröm and Litzén [10]. For the resonance ${ }^{1} \mathrm{~S}_{0} \rightarrow 2 \mathrm{p}^{1} \mathrm{P}_{1}$ transition, it is seen that the current SCUNC results compared very well to the experimental values. Here, the $\Delta \lambda / \lambda$ percentage deviations with 
Table 1. Present wavelengths $(\lambda$, in $\AA)$ of the $1 \mathrm{~s}^{2} \mathrm{~S}_{0} \rightarrow 1 \mathrm{~s} n \mathrm{p}{ }^{1} \mathrm{P}_{1}$ transitions in He-like $(Z=3-15)$ ions.

\begin{tabular}{|c|c|c|c|c|c|c|c|c|c|c|c|c|c|}
\hline $1 \mathrm{~S}-n^{1} \mathrm{P}$ & $\begin{array}{c}\mathrm{Li} \text { II } \\
\lambda\end{array}$ & $\begin{array}{c}\text { Be III } \\
\lambda\end{array}$ & $\begin{array}{c}\text { B IV } \\
\lambda\end{array}$ & $\begin{array}{c}\mathrm{C} V \\
\lambda\end{array}$ & $\begin{array}{c}\text { N VI } \\
\lambda\end{array}$ & $\begin{array}{c}\text { O VII } \\
\lambda\end{array}$ & $\begin{array}{c}\text { F VIII } \\
\lambda\end{array}$ & $\begin{array}{c}\text { Ne IX } \\
\lambda\end{array}$ & $\begin{array}{c}\mathrm{Na} X \\
\lambda\end{array}$ & $\underset{\lambda}{\mathrm{Mg} \mathrm{XI}}$ & $\begin{array}{c}\mathrm{Al} \text { XII } \\
\lambda\end{array}$ & $\begin{array}{c}\text { Si XIII } \\
\lambda\end{array}$ & $\begin{array}{c}\mathrm{P} X I V \\
\lambda\end{array}$ \\
\hline $1 \mathrm{~S}-3^{1} \mathrm{P}$ & 178.0140 & 88.3140 & 52.6852 & 34.9749 & 24.9012 & 18.6283 & 14.4588 & 11.5474 & 9.4344 & 7.8524 & 6.6374 & 5.6841 & 4.9222 \\
\hline $1 \mathrm{~S}-4^{1} \mathrm{P}$ & 171.5750 & 84.7502 & 50.4334 & 33.4271 & 23.7736 & 17.7709 & 13.7853 & 11.5474 & 9.4344 & 7.8524 & 6.6374 & 5.6841 & 4.9222 \\
\hline $1 \mathrm{~S}-5^{1} \mathrm{P}$ & 168.7422 & 83.1934 & 49.4536 & 32.7553 & 23.2850 & 17.3999 & 13.4942 & 11.0046 & 8.9877 & 7.4785 & 6.3199 & 5.4110 & 4.6850 \\
\hline $1 S-6^{1} \mathrm{P}$ & 167.2401 & 82.3706 & 48.9368 & 32.4014 & 23.0278 & 17.2047 & 13.348 & 10.7701 & 8.7949 & 7.3171 & 6.1829 & 5.2933 & 4.5827 \\
\hline $1 S-7^{1} \mathrm{P}$ & 166.3466 & 81.8821 & 48.6302 & 32.1916 & 22.8754 & 17.0891 & 13.2504 & 10.5738 & 8.6335 & 7.1821 & 6.0683 & 5.1948 & 4.4972 \\
\hline $1 \mathrm{~S}-8^{1} \mathrm{P}$ & 165.7714 & 81.5679 & 48.4332 & 32.0568 & 22.7775 & 17.0148 & 13.1922 & 10.5270 & 8.5950 & 7.1499 & 6.0409 & 5.1713 & 4.4768 \\
\hline $1 \mathrm{~S}-9^{1} \mathrm{P}$ & 165.3792 & 81.3539 & 48.2990 & 31.9651 & 22.789 & 16.9643 & 13.1525 & 10.4951 & 8.5688 & 7.1280 & 6.0223 & 5.1553 & 4.4629 \\
\hline $1 \mathrm{~S}-10^{1} \mathrm{P}$ & 165.0997 & 81.2015 & 48.2035 & 31.8997 & 22.6635 & 16.9284 & 13.1243 & 10.4724 & 8.5501 & 7.1124 & 6.0091 & 5.1440 & 4.4531 \\
\hline $1 \mathrm{~S}-11^{1} \mathrm{P}$ & 164.8934 & 81.0890 & 48.1331 & 31.8516 & 22.6285 & 16.9018 & 13.836 & 10.4557 & 8.5364 & 7.1009 & 5.9993 & 5.1356 & 4.4458 \\
\hline $1 \mathrm{~S}-12^{1} \mathrm{P}$ & 1647369 & 81.0037 & 48.0796 & 31.8150 & 22.6020 & 16.8817 & 13.0878 & 10.4430 & 8.5259 & 7.0922 & 5.9919 & 5.1292 & 4.4403 \\
\hline $1 \mathrm{~S}-13^{1} \mathrm{P}$ & 164.6153 & 80.9374 & 48.0381 & 31.7867 & 22.5814 & 16.8661 & 13.0756 & 10.4331 & 8.5178 & 7.0854 & 5.9862 & 5.1243 & 4.4360 \\
\hline $1 \mathrm{~S}-14^{1} \mathrm{P}$ & 164.5187 & 80.8849 & 48.0052 & 31.7642 & 22.5651 & 16.8537 & 13.0659 & 10.4253 & 8.5114 & 7.0801 & 5.9816 & 5.1204 & 4.4326 \\
\hline $1 \mathrm{~S}-15^{1} \mathrm{P}$ & 164.4410 & 80.8425 & 47.9787 & 31.7461 & 22.5519 & 16.8438 & 13.0580 & 10.4190 & 8.5063 & 7.0757 & 5.9780 & 5.1172 & 4.4298 \\
\hline $1 \mathrm{~S}-16^{1} \mathrm{P}$ & 164.3775 & 80.8079 & 47.9570 & 31.7312 & 22.5412 & 16.8356 & 13.0517 & 10.4139 & 8.5020 & 7.0722 & 5.9750 & 5.1147 & 4.4276 \\
\hline $1 \mathrm{~S}-17^{1} \mathrm{P}$ & 164.3248 & 80.7793 & 47.9391 & 31.7190 & 22.5323 & 16.8289 & 13.0464 & 10.4096 & 8.4985 & 7.0693 & 5.9725 & 5.1125 & 4.4258 \\
\hline $1 \mathrm{~S}-18^{1} \mathrm{P}$ & 164.2808 & 80.7553 & 47.9240 & 31.7087 & 22.5248 & 16.8232 & 13.0419 & 10.4061 & 8.4956 & 7.0668 & 5.9704 & 5.1107 & 4.4242 \\
\hline $1 \mathrm{~S}-19^{1} \mathrm{P}$ & 164.2435 & 80.7349 & 47.9113 & 31.7000 & 22.5185 & 16.8184 & 13.0382 & 10.4030 & 8.4931 & 7.0648 & 5.9687 & 5.1092 & 4.4229 \\
\hline $1 \mathrm{~S}-20^{1} \mathrm{P}$ & 164.2117 & 80.7176 & 47.9005 & 31.6926 & 22.5131 & 16.8144 & 13.0350 & 10.4005 & 8.4910 & 7.0630 & 5.9672 & 5.1079 & 4.4218 \\
\hline $1 \mathrm{~S}-21^{1} \mathrm{P}$ & 164.1843 & 80.7027 & 47.8911 & 31.6862 & 22.5085 & 16.8109 & 13.0322 & 10.3983 & 8.4892 & 7.0615 & 5.9659 & 5.1068 & 4.4208 \\
\hline $1 \mathrm{~S}-22^{1} \mathrm{P}$ & 164.1605 & 80.6898 & 47.8831 & 31.6807 & 22.5045 & 16.8078 & 13.0299 & 10.3963 & 8.4876 & 7.0602 & 5.9648 & 5.1059 & 4.4200 \\
\hline $1 \mathrm{~S}-23^{1} \mathrm{P}$ & 164.1399 & 80.6785 & 47.8760 & 31.6759 & 22.5010 & 16.8052 & 13.0278 & 10.3947 & 8.4862 & 7.0590 & 5.9638 & 5.1050 & 4.4193 \\
\hline $1 \mathrm{~S}-24^{1} \mathrm{P}$ & 164.1217 & 80.6686 & 47.8698 & 31.6717 & 22.4979 & 16.8028 & 13.0260 & 10.3932 & 8.4850 & 7.0580 & 5.9629 & 5.1043 & 4.4186 \\
\hline $1 \mathrm{~S}-25^{1} \mathrm{P}$ & 164.1057 & 80.6599 & 47.8644 & 31.6679 & 22.4952 & 16.8008 & 13.0243 & 10.3919 & 8.4840 & 7.0571 & 5.9622 & 5.1036 & 4.4181 \\
\hline
\end{tabular}

Table 2. Theoretical and experimental wavelengths of the $1{ }^{1} \mathrm{~S}_{0} \rightarrow n \mathrm{p}{ }^{1} \mathrm{P}_{1}\left(1 \mathrm{~s}^{2}{ }^{1} \mathrm{~S}_{0} \rightarrow 1 \mathrm{~s} n \mathrm{p}{ }^{1} \mathrm{P}_{1}\right)$ transitions of helium-like ions up to $Z$ $=8$.

\begin{tabular}{|c|c|c|c|c|c|c|c|c|c|}
\hline \multirow{2}{*}{$1 S-n^{1} P$} & \multicolumn{3}{|c|}{ Li II } & \multicolumn{3}{|c|}{ Be III } & \multicolumn{3}{|c|}{ B IV } \\
\hline & $\lambda^{\mathrm{P}}$ & $\lambda \exp ^{(a)}$ & $\Delta \lambda / \lambda$ & $\lambda^{\mathrm{p}}$ & $\lambda \exp ^{(a)}$ & $\Delta \lambda / \lambda$ & $\lambda^{\mathrm{p}}$ & $\lambda \exp ^{(\mathrm{a})}$ & $\Delta \lambda / \lambda$ \\
\hline $1 \mathrm{~S}-2^{1} \mathrm{P}$ & 199.2800 & 199.280 & $0.0000 \%$ & 80.2522 & 80.254 & $0.0018 \%$ & 60.390 & 60.313 & $0.0033 \%$ \\
\hline $1 S-3^{1} \mathrm{P}$ & 178.0140 & 178.014 & $0.0000 \%$ & 88.3140 & 88.314 & $0.0000 \%$ & 52.6852 & 52.679 & $0.098 \%$ \\
\hline $1 \mathrm{~S}-4^{1} \mathrm{P}$ & 171.5750 & 171.575 & $0.0000 \%$ & 84.7502 & 84.758 & $0.0092 \%$ & 50.4334 & 50.435 & $0.0032 \%$ \\
\hline $1 S-5^{1} \mathrm{P}$ & 168.7421 & & & 83.1934 & 83.202 & $0.083 \%$ & 49.4536 & 49.456 & $0.0048 \%$ \\
\hline $1 \mathrm{~S}-6^{1} \mathrm{P}$ & 167.2401 & & & 82.3706 & 82.377 & $0.0198 \%$ & 48.9368 & & \\
\hline $1 \mathrm{~S}-7^{1} \mathrm{P}$ & 166.3466 & & & 81.8821 & 81.891 & $0.089 \%$ & 48.6302 & & \\
\hline $1 S-8^{1} \mathrm{P}$ & 165.7714 & & & 81.5679 & & & 48.4332 & & \\
\hline $1 S-9^{1} \mathrm{P}$ & 165.3792 & & & 81.3539 & & & 48.2990 & & \\
\hline $1 \mathrm{~S}-8^{1} \mathrm{P}$ & 165.0997 & & & 81.2015 & & & 48.2035 & & \\
\hline
\end{tabular}


Continued

\begin{tabular}{|c|c|c|c|c|c|c|c|c|c|}
\hline \multirow{2}{*}{$1 S-n^{1} P$} & \multicolumn{3}{|c|}{$\mathrm{CV}$} & \multicolumn{3}{|c|}{ NVI } & \multicolumn{3}{|c|}{ OVII } \\
\hline & $\lambda n r^{p}$ & $\lambda \exp ^{(a, b)}$ & $\Delta \lambda / \lambda^{*}$ & $\lambda n r^{p}$ & $\lambda \exp ^{(d)}$ & $\Delta \lambda / \lambda$ & $\lambda \mathrm{nr}^{\mathrm{p}}$ & $\lambda \exp ^{(a, c)}$ & \\
\hline $1 \mathrm{~S}-2^{1} \mathrm{P}$ & 40.2647 & $40.268^{\mathrm{b}}$ & $0.0082 \%$ & 28.7857 & 28.787 & $0.0045 \%$ & 21.6021 & $21.602^{\mathrm{a}}$ & $0.0005 \%$ \\
\hline $1 \mathrm{~S}-3^{1} \mathrm{P}$ & 34.9749 & $34.973^{\mathrm{a}, \mathrm{b}}$ & $0.0054 \%$ & 24.9012 & 24.898 & $0.0128 \%$ & 18.6283 & & \\
\hline $1 \mathrm{~S}-4^{1} \mathrm{P}$ & 33.4271 & $33.426^{\mathrm{a}, \mathrm{b}}$ & $0.0033 \%$ & 23.7736 & 23.771 & $0.089 \%$ & 17.7709 & & \\
\hline $1 \mathrm{~S}-5^{1} \mathrm{P}$ & 32.7553 & $32.754^{\mathrm{a}, \mathrm{b}}$ & $0.0039 \%$ & 23.2850 & 23.281 & $0.0172 \%$ & 17.3999 & & \\
\hline $1 S-6^{1} \mathrm{P}$ & 32.4014 & $32.399^{\mathrm{b}}$ & $0.0074 \%$ & 23.0278 & 23.024 & $0.0165 \%$ & 17.2047 & $17.199^{c}$ & $0.0331 \%$ \\
\hline $1 \mathrm{~S}-7^{1} \mathrm{P}$ & 32.1916 & & & 22.8754 & & & 17.0891 & $17.083^{c}$ & $0.0357 \%$ \\
\hline $1 \mathrm{~S}-8^{1} \mathrm{P}$ & 32.0568 & & & 22.7775 & & & 17.0148 & $17.008^{c}$ & $0.0399 \%$ \\
\hline $1 \mathrm{~S}-9^{1} \mathrm{P}$ & 31.9651 & & & 22.789 & & & 16.9643 & $16.957^{\mathrm{c}}$ & $0.0431 \%$ \\
\hline $1 \mathrm{~S}-8^{1} \mathrm{P}$ & 31.8997 & & & 22.6635 & & & 16.9284 & $16.924^{\mathrm{c}}$ & $0.0230 \%$ \\
\hline
\end{tabular}

Here, $\lambda^{\mathrm{p}}$ denotes the present SCUNC calculations values, $\lambda$ exp represents the experimental values and $\Delta \lambda / \lambda$ stands for the percentage deviations with respect to the experimental value of the corresponding system. (a), experimental data of Robinson [7]; (b), experimental data of Svensson [8]; (c), experimental data of Bartnik et al. [11]; (d), experimental data of Engtröm and Litzén [10]. Wavelengths are in angstroms.

respect to the experimental values of the corresponding system are less than $0.009 \%$. The slight discrepancies can be explained by the fact that the present formalism disregards explicitly mass polarization, relativistic and QED corrections. For the transitions $1{ }^{1} \mathrm{~S}_{0} \rightarrow n \mathrm{p}^{1} \mathrm{P}_{1}(n \geq 3)$, comparison with the quoted experimental data indicates again good agreements. For these levels, the percentage deviations with respect to the experimental value of the corresponding system are less than $0.05 \%$. Here, the discrepancies may be imputed mainly to mass polarization corrections which are not taken into account in the present calculations. In fact, and as well mentioned by Beiersdorfer et al. [9], the $n \geq 3$ levels are less affected by electron-electron interactions, relativistic and QED corrections. Then, for $n \geq 3$ states, the ratio $m / M$ ( $m$ and $M$ respectively the electron and nuclear masses) becomes important while increasing the $Z$-charge number. Nevertheless, the present SCUNC semi-empirical formulas may be considered as good representative of experimental data when electron-electron interactions, relativistic and QED corrections are disregarded. In Table 3, the SCUNC predictions for the wavelengths belonging to the $1 \mathrm{~s}^{2}{ }^{1} \mathrm{~S}_{0} \rightarrow 1 \mathrm{~s} 2 \mathrm{p}{ }^{1,3} \mathrm{P}_{1}$ transitions in He-like ions are compared to the ab initio calculations of Acaad et al., [12] using wave function expanded in a triple series of Laguerre polynomials of the perimertric coordinates, the computational results of Safronova et al., [13] applying the MZ code through a perturbation theory based on hydrogen-like functions and with the data of Porter [14] using the plasma simulation code CLOUDY. The overall agreement between the calculations is reasonably gratifying. Here, the $\left|\Delta \lambda_{\text {theo }}\right|$ differences in wavelengths between the present calculations and the theoretical literature data [12] [13] [15] have never overrun $0.003 \AA$ for the $1 \mathrm{~s}^{2} \mathrm{~S}_{0} \rightarrow 1 \mathrm{~s} 2 \mathrm{p}{ }^{1} \mathrm{P}_{1}$ resonance line and $0.008 \AA$ for the $1 \mathrm{~s}^{2}{ }^{1} \mathrm{~S}_{0} \rightarrow 1 \mathrm{~s} 2 \mathrm{p}{ }^{3} \mathrm{P}_{1}$ intercombination line up to $Z=22$. This may point out 
Table 3. Theoretical wavelengths for $1 \mathrm{~s}^{2}{ }^{1} \mathrm{~S}_{0} \rightarrow 1 \mathrm{~s} 2 \mathrm{p}^{1,3} \mathrm{P}_{1}$ for He-like ions $(2 \leq Z \leq 22)$.

\begin{tabular}{|c|c|c|c|c|c|c|c|c|c|c|}
\hline \multirow{2}{*}{$Z$} & \multicolumn{5}{|c|}{$1 s^{2}{ }^{1} S_{0} \rightarrow 1 s 2 p^{1} P_{1}$ (resonance line: $r$ ) } & \multicolumn{5}{|c|}{$1 \mathrm{~s}^{2} \mathrm{~S}_{0} \rightarrow 1 \mathrm{~s} 2 \mathrm{p}^{3} \mathrm{P}_{1}$ (intercombinaison line: 1 ) } \\
\hline & $\lambda p$ & $\lambda$ theo $^{\mathrm{a}, \mathrm{b}}$ & $\lambda$ theo $^{c}$ & $\mid \Delta \lambda$ theo $\left.\right|^{\mathrm{a}, \mathrm{b}}$ & $\mid \Delta \lambda$ theo $\left.\right|^{c}$ & $\lambda p$ & $\lambda$ theo $o^{a, b}$ & $\lambda$ theo $^{c}$ & $\mid \Delta \lambda$ theo $\left.\right|^{\mathrm{a}, \mathrm{b}}$ & $\mid \Delta \lambda$ theo $\left.\right|^{\circ}$ \\
\hline 2 & 584.3339 & $584.3343^{\mathrm{a}}$ & & 0.0004 & & 591.4121 & $591.499^{\mathrm{a}}$ & & 0.0002 & \\
\hline 3 & 199.2800 & $199.2791^{a}$ & & 0.0009 & & 202.2252 & & & & \\
\hline 4 & 80.2522 & $80.2535^{\mathrm{a}}$ & & 0.0013 & & 81.6677 & & & & \\
\hline 5 & 60.390 & $60.3135^{\mathrm{a}}$ & & 0.0020 & & 61.0880 & $61.0882^{\mathrm{a}}$ & & 0.0002 & \\
\hline 6 & 40.2647 & $40.2671^{\mathrm{a}}$ & 40.2680 & 0.0024 & 0.0033 & 40.7302 & $40.7299^{\mathrm{a}}$ & 40.7310 & 0.0003 & 0.0008 \\
\hline 7 & 28.7857 & $28.7867^{\mathrm{a}}$ & 28.7870 & 0.0010 & 0.0013 & 29.0818 & $29.0840^{\mathrm{a}}$ & 29.0840 & 0.0022 & 0.0022 \\
\hline 8 & 21.6021 & $21.6012^{\mathrm{a}}$ & 21.6020 & 0.0009 & 0.0001 & 21.7988 & $21.8033^{\mathrm{a}}$ & 21.8070 & 0.0008 & 0.0045 \\
\hline 9 & 16.8088 & $16.8061^{\mathrm{a}}$ & 16.8070 & 0.0027 & 0.0018 & 16.9438 & $16.9496^{\mathrm{a}}$ & 16.9470 & 0.0045 & 0.0082 \\
\hline 8 & 13.4514 & & 13.4470 & & 0.0044 & 13.5464 & & 13.5530 & & 0.0066 \\
\hline 9 & 9.0050 & & 9.0030 & & 0.0020 & 9.0880 & & 9.0830 & & 0.0060 \\
\hline 12 & 9.1689 & & 9.1688 & & 0.0001 & 9.2310 & & 9.2312 & & 0.0062 \\
\hline 13 & 7.7568 & & 7.7573 & & 0.0005 & 7.8044 & & 7.8070 & & 0.0026 \\
\hline 14 & 6.6475 & & 6.6480 & & 0.0005 & 6.6847 & & 6.6883 & & 0.0036 \\
\hline 15 & 5.7701 & $50386^{\mathrm{b}}$ & & & & 5.7898 & $50667^{b}$ & & & \\
\hline 16 & 5.0387 & & 5.0387 & 0.0002 & 0.0000 & 5.0667 & & 5.0665 & 0.0000 & 0.0002 \\
\hline 17 & 4.4445 & $4.4445^{\mathrm{b}}$ & & 0.0002 & & 4.4682 & $4.4681^{\mathrm{b}}$ & & 0.0001 & \\
\hline 18 & 3.9491 & $3.9492^{\mathrm{b}}$ & 3.9488 & 0.0001 & 0.0003 & 3.9694 & $3.9695^{\mathrm{b}}$ & 3.9691 & 0.0001 & 0.0003 \\
\hline 19 & 3.5318 & $3.5319^{\mathrm{b}}$ & & 0.0002 & & 3.5493 & & & & \\
\hline 20 & 3.1771 & $3.1772^{\mathrm{b}}$ & 3.1772 & 0.0001 & 0.0001 & 3.1924 & $3.1928^{\mathrm{b}}$ & 3.1928 & 0.0004 & 0.0004 \\
\hline 21 & 2.8731 & $2.8731^{\mathrm{b}}$ & & 0.0000 & & 2.8866 & $2.8871^{\mathrm{b}}$ & & 0.0005 & \\
\hline 22 & 2.684 & $2.685^{\mathrm{b}}$ & & 0.0001 & & 2.6226 & $2.6230^{\mathrm{b}}$ & & 0.0004 & \\
\hline
\end{tabular}

Here, $\lambda^{\mathrm{p}}$ denotes the present SCUNC calculations, $\lambda$ theo represents the theoretical values and $\mid \Delta \lambda$ theo $\mid$ stands for the difference in wavelengths between the present calculations and the other theoretical ones $\left(\lambda\right.$ theo $^{\mathrm{a}}$ or $\lambda$ theo $\left.^{\mathrm{b}}\right)$. (a): calculations of Accad et al., [12], (b): calculations of Safronova et al. [13]; (c): calculations of Porter [14]. Wavelengths are in angstroms.

the good agreement between the calculations. The discrepancies with respect to the accurate $a b$ initio computations are due to the present none-relativistic formalism. Table 4, shows a comparison of the present wavelengths for the forbidden $1 \mathrm{~s}^{2} \mathrm{~S}_{0} \rightarrow 1 \mathrm{~s} 2 \mathrm{~s}{ }^{3} \mathrm{~S}_{1}$ transitions of He-like systems $(Z=2-15)$ with the NIST compiled data. Excellent agreement is obtained between the SCUNC predictions and the NIST data. Except for $Z=8$, the maximum shift in wavelengths with respect to the NIST values is at $0.003 \AA$. In Table 5, the present theoretical wavelengths for the $1 \mathrm{~s} n \mathrm{p}{ }^{1} \mathrm{P} 1 \rightarrow 1 \mathrm{~s}^{2}{ }^{1} \mathrm{~S} 0(2 \leq n \leq 5)$ transitions of the helium-like ions up to $Z=9$ are compared to the $\lambda$ nrel-nonrelativistic wavelengths values and to the $\lambda_{\text {tot }}$-total wavelengths (including mass polarization, relativistic corrections and the Lamb-shift correction for the $1{ }^{1} \mathrm{~S}$ level) computed by Accad et al. [12]. For the $1 \mathrm{~s}^{2}{ }^{1} \mathrm{~S}_{0} \rightarrow 1 \mathrm{~s} 2 \mathrm{p}{ }^{1} \mathrm{P}_{1}$ resonance line, the uncertainties between the present calculations and the $\lambda_{\text {tot }}-$ total wavelengths 
Table 4. Comparison of the SCUNC predictions with the NIST data the wavelengths belonging to the forbidden $1 \mathrm{~s}^{2}{ }^{1} \mathrm{~S}_{0} \rightarrow 1 \mathrm{~s} 2 \mathrm{~s}^{3} \mathrm{~S}_{1}$ transitions in He-like $(Z=2-15)$ systems. Wavelengths are in angstroms.

\begin{tabular}{cccc}
\hline$Z$ & $\lambda$ SCUNC & $\lambda$ NIST & $|\Delta \lambda|^{*}$ \\
\hline 2 & 625.563 & 625.563 & 0.000 \\
3 & 210.069 & 210.069 & 0.000 \\
4 & 104.547 & 104.548 & 0.001 \\
5 & 62.439 & 62.440 & 0.001 \\
6 & 41.469 & 41.472 & 0.003 \\
7 & 29.531 & 29.534 & 0.003 \\
8 & 22.094 & 22.101 & 0.007 \\
9 & 17.149 & - & 0.003 \\
10 & 13.696 & 13.699 & 0.002 \\
11 & 11.190 & 11.192 & \\
12 & 9.313 & - & \\
13 & 7.872 & - & 0.001 \\
14 & 6.741 & 6.740 & \\
15 & 5.838 & - & \\
\hline
\end{tabular}

$*|\Delta \lambda|=\left|\lambda^{\mathrm{SCUNC}}-\right| \lambda^{\mathrm{NIST}} \mid$.

Table 5. Theoretical wavelengths for the $1 \mathrm{~s}^{2}{ }^{1} \mathrm{~S}_{0} \rightarrow 1 \mathrm{~s} n \mathrm{p}{ }^{1} \mathrm{P}_{1}(2 \leq n \leq 5)$ transitions in He-like $(Z=3$ - 9) ions. Here, $\lambda$ denotes the present SCUNC calculations, $\lambda$ nrel denotes the nonrelativistic wavelengths and $\lambda$ tot the theoretical wavelengths of Accad et al. [12] including mass polarization, relativistic corrections and the Lamb-shift correction for the $1 \mathrm{~S}$ level. Wavelengths are in angstroms.

\begin{tabular}{|c|c|c|c|c|c|c|}
\hline \multirow[b]{2}{*}{ System } & \multirow[b]{2}{*}{ Transition } & \multicolumn{3}{|c|}{ Theory } & \multicolumn{2}{|c|}{ Comparison } \\
\hline & & $\begin{array}{c}\text { Present } \\
\lambda\end{array}$ & $\begin{array}{c}\text { Accad et al. } \\
\lambda \text { nrel }\end{array}$ & $\begin{array}{c}\text { Accad et al. } \\
\lambda \text { tot }\end{array}$ & $\mid \lambda-\lambda$ nrel $\mid$ & $\mid \lambda-\lambda$ tot $\mid$ \\
\hline \multirow{5}{*}{ Li II } & $1 S-2^{1} \mathrm{P}$ & 199.2800 & 199.2813 & 199.2791 & 0.0013 & 0.0009 \\
\hline & $1 S-3^{1} \mathrm{P}$ & 178.0140 & 178.0162 & 178.0143 & 0.0022 & 0.0003 \\
\hline & $1 \mathrm{~S}-4^{1} \mathrm{P}$ & 171.5750 & 171.5776 & 171.5757 & 0.0026 & 0.0007 \\
\hline & $1 \mathrm{~S}-5^{1} \mathrm{P}$ & 168.7421 & & & & \\
\hline & $1 S-2^{1} \mathrm{P}$ & 80.2522 & 80.2600 & 80.2535 & 0.0078 & 0.0013 \\
\hline \multirow[t]{4}{*}{ Be III } & $1 S-3^{1} \mathrm{P}$ & 88.3140 & 88.3134 & 88.3075 & 0.0006 & 0.0065 \\
\hline & $1 \mathrm{~S}-4^{1} \mathrm{P}$ & 84.7502 & 84.7588 & 84.7532 & 0.0086 & 0.0030 \\
\hline & $1 \mathrm{~S}-5^{1} \mathrm{P}$ & 83.1934 & 83.2044 & 83.1989 & 0.090 & 0.0055 \\
\hline & $1 S-2^{1} \mathrm{P}$ & 60.390 & 60.3224 & 60.3135 & 0.094 & 0.0025 \\
\hline \multirow[t]{4}{*}{ B IV } & $1 \mathrm{~S}-3^{1} \mathrm{P}$ & 52.6852 & 52.6876 & 52.6800 & 0.0024 & 0.0052 \\
\hline & $1 \mathrm{~S}-4^{1} \mathrm{P}$ & 50.4334 & 50.4408 & 50.4335 & 0.0074 & 0.0001 \\
\hline & $1 S-5^{1} \mathrm{P}$ & 49.4536 & 49.4621 & 49.4549 & 0.0085 & 0.0013 \\
\hline & $1 \mathrm{~S}-2^{1} \mathrm{P}$ & 40.2647 & 40.2774 & 40.2671 & 0.0127 & 0.0024 \\
\hline
\end{tabular}




\begin{tabular}{|c|c|c|c|c|c|c|}
\hline \multicolumn{7}{|c|}{ Continued } \\
\hline \multirow[t]{4}{*}{$\mathrm{CV}$} & $1 S-3^{1} \mathrm{P}$ & 34.9749 & 34.9811 & 34.9723 & 0.0062 & 0.0026 \\
\hline & $1 \mathrm{~S}-4^{1} \mathrm{P}$ & 33.4271 & 33.4343 & 33.4259 & 0.0072 & 0.0012 \\
\hline & $1 S-5^{1} \mathrm{P}$ & 32.7553 & 32.7622 & 32.7540 & 0.0069 & 0.0013 \\
\hline & $1 S-2^{1} \mathrm{P}$ & 28.7857 & 28.7980 & 28.7867 & 0.0123 & 0.0010 \\
\hline \multirow[t]{4}{*}{ N VI } & $1 S-3^{1} \mathrm{P}$ & 24.9012 & 24.9098 & 24.9002 & 0.0086 & 0.0010 \\
\hline & $1 \mathrm{~S}-4^{1} \mathrm{P}$ & 23.7736 & 23.7806 & 23.7714 & 0.0070 & 0.0022 \\
\hline & $1 \mathrm{~S}-5^{1} \mathrm{P}$ & 23.2850 & & & & \\
\hline & $\begin{array}{l}1 S-2^{1} \mathrm{P} \\
1 S-3^{1} \mathrm{P}\end{array}$ & 21.6021 & 21.6133 & 21.6012 & 0.092 & 0.0009 \\
\hline \multirow[t]{4}{*}{ O VII } & & 18.6283 & 18.6381 & 18.6280 & 0.0098 & 0.0003 \\
\hline & $1 \mathrm{~S}-4^{1} \mathrm{P}$ & 17.7709 & 17.7777 & 17.7680 & 0.0068 & 0.0029 \\
\hline & $1 S-5^{1} \mathrm{P}$ & 17.3999 & 17.4051 & 17.3957 & 0.0052 & 0.0042 \\
\hline & $1 S-2^{1} \mathrm{P}$ & 16.8088 & 16.8188 & 16.8061 & 0.080 & 0.0027 \\
\hline \multirow[t]{3}{*}{ F VIII } & $1 \mathrm{~S}-3^{1} \mathrm{P}$ & 14.4588 & 14.4690 & 14.4584 & 0.082 & 0.0004 \\
\hline & $1 \mathrm{~S}-4^{1} \mathrm{P}$ & 13.7853 & & & & \\
\hline & $1 S-5^{1} \mathrm{P}$ & 13.4942 & & & & \\
\hline
\end{tabular}

results [12] are less than $0.003 \AA$. As far as comparison with the $\lambda_{\text {nrel }}$-nonrelativistic wavelengths values are concerned, it is seen that the uncertainties are about $0.01 \AA$ for $Z=5$ - 9. This points out that, the present SCUNC results are most accurate than the $\lambda_{\text {nrel }}$-nonrelativistic wavelengths obtained by Accad et al. [12] when increasing the nuclear charge. For $n \geq 3$ states, it can also be seen that the present SCUNC wavelengths values are most accurate than that of Accad et al. [12]. Here, the uncertainties with respect to the $\lambda_{\text {tot }}$-total wavelengths are less than $0.005 \AA$ for all the entire series considered $(Z=2$ - 9) whereas the uncertainties with respect to the $\lambda_{\text {nrel }}$-nonrelativistic wavelengths increase up to $0.01 \AA$ for $Z=9$. This may point out again that, in the SCUNC formalism, relativistic effects are implicitly incorporated in the $f$-screening constants evaluated from experimental data. Besides, it should be mentioned that the $\lambda_{\text {tot }}$-total wavelengths equal to $88.3075 \AA$ for the $1 \mathrm{~s}^{2}{ }^{1} \mathrm{~S}_{0} \rightarrow 1 \mathrm{~s} 3 \mathrm{p}{ }^{1} \mathrm{P}_{1}$ transition of Be III may be probably lower as the corresponding high precision measurement is at $88.3140 \AA$ [7] to be compared to the present prediction at $88.3140 \AA$ A.

\section{Conclusion}

The Screening Constant per Unit Nuclear Charge method has been applied to inaugurate the first spectral lines for the three most intense lines (resonance line $1 \mathrm{~s}^{2} \mathrm{~S}_{0}-1 \mathrm{~s} 2 \mathrm{p}^{1} \mathrm{P}_{1}$ intercombination line $1 \mathrm{~s}^{2}{ }^{1} \mathrm{~S}_{0}-1 \mathrm{~s} 2 \mathrm{p}^{3} \mathrm{P}_{1}$ and forbidden line $1 \mathrm{~s}^{2} \mathrm{~S}_{0}$ - $1 \mathrm{~s} 2 \mathrm{~s}^{3} \mathrm{~S}_{1}$ and for the $1 \mathrm{~s}^{2} \mathrm{~S}_{0}-1 \mathrm{~s} n \mathrm{p}^{1} \mathrm{P}_{1}$ transitions in the helium isoelectronic sequence. In our knowledge, only the spectral lines of the Hydrogen-like ions have 
determined empirically in the past. At present hour, the possibilities to calculate easily the most intense lines of helium-like systems in the X-ray range in connection with plasma diagnostic are demonstrated in this work. All the results obtained in the present paper compared very well to various experimental and theoretical literature data. It should be underlined the merit of the SCUNC formalism providing accurate results via simple analytical formulas without needing to use codes of simulation. The accurate results obtained in this work point out the possibilities to investigate highly charged He-positive like ions in the framework of the SCUNC method.

\section{Conflicts of Interest}

The author declares no conflicts of interest regarding the publication of this paper.

\section{References}

[1] Gabriel, A. and Jordan, C. (1969) Monthly Notices of the Royal Astronomical Society, 145, 241-248. https://doi.org/10.1093/mnras/145.2.241

[2] Keenan, F.P., McCann, S.M., Kingstne, A.E. and McKenzie, D.L. (1987) Astrophysical Journal, 318, 926-929. https://doi.org/10.1086/165424

[3] McKenzie, D.L. and Landecker, P.B. (1982) Astrophysical Journal, 259, 372-380. https://doi.org/10.1086/160174

[4] Porquet, D. and Dubau, J. (2000) Astronomy and Astrophysics Supplement, 143, 495-514. https://doi.org/10.1051/aas:2000192

[5] Liedahl, D.A. (1999) X-Ray Spectroscopy in Astrophysics, EADN School Proceedings, 1997, In: Van Paradijs, J.A. and Bleeker, J.A.M., Eds., 189.

[6] Porquet, D., Mewe, R., Raassen, A.J.J., Kaastra, J.S. and Dubau, J. (2001) Helium-Like Ions as Powerful X-Ray Plasma Diagnostics. X-Ray Astronomy 2000. ASP Conference Proceeding, Vol. 234, 121-127.

[7] Robinson, H.A. (1937) Physical Review, 14, 51. https://doi.org/10.1103/PhysRev.51.14

[8] Svensson, L.A. (1970) Physica Scripta, 1, 246. https://doi.org/10.1088/0031-8949/1/5-6/009

[9] Beiersdorfer, P., Bitter, M., von Goeler, S. and Hill, K.W. (1989) Physical Review A, 40, 150. https://doi.org/10.1103/PhysRevA.40.150

[10] Engström, L. and litzén, U. (1995) Journal of Physics B: Atomic, Molecular and Optical Physics, 28, 2565. https://doi.org/10.1088/0953-4075/28/13/010

[11] Bartnik, A.E., Biémont, E., Dyakin, V.M., Ya Faenov, A., Fiedorowicz, H., et al. (1997) Journal of Physics B: Atomic, Molecular and Optical Physics, 30, 4453. https://doi.org/10.1088/0953-4075/30/20/009

[12] Accad, Y., Pekeris, C.L. and Schiff, B. (1971) Physical Review A, 4, 516. https://doi.org/10.1103/PhysRevA.4.516

[13] Safronova, U.I., Safronova, M.S. and Bruch, R. (1995) Journal of Physics B: Atomic, Molecular and Optical Physics, 28, 2803. https://doi.org/10.1088/0953-4075/28/14/005

[14] Porter, R.L. (2006) Theory and Application of Helium and Helium-Like Ions in As- 
trophysical Environments. College of Art and Sciences, Lexington University of Kentucky, Lexington.

[15] Sakho, I. (2017) Atomic Data Nuclear Data Tables, 97, 425-438. https://doi.org/10.1016/j.adt.2016.12.001

[16] Sakho, I. (2018) Journal of Electron Spectroscopy and Related Phenomena, 222, 40-50. https://doi.org/10.1016/j.elspec.2017.09.011

[17] Radzig, A.A. and Smirnov, M.B. (1985) Reference Data on Atoms, Molecules and Ions (Berlin: Springer) Moore E C 1971 Atomic Energy Levels (Natl. Stand. Ref. Data Ser. Natl. Bur. Stand. No. 35). U.S. GPO, Washington DC, Vol. 1, 4.

[18] Arnaud, P. (1993) Cours de chimie physique. 3rd Edition, Dunod, Paris, Chapter 11,88 .

[19] Herzberg, G. (1958) Proceedings of the Royal Society (London) A, 248, 309-332. https://doi.org/10.1098/rspa.1958.0246

[20] Kramida, A., Ralchenko, Y., Reader, J. and NIST ASD Team (2018) NIST Atomic Spectra Database (ver. 5.6.1). National Institute of Standards and Technology, Gaithersburg. https://physics.nist.gov/asd 